\title{
Validation of the Caprini Risk Assessment Model in Plastic and Reconstructive Surgery Patients
}

\author{
Christopher J. Pannucci, MD, MS, \\ Section of Plastic Surgery, University of Michigan, Ann Arbor, MI \\ Steven H. Bailey, MD, \\ Department of Plastic Surgery, University of Texas-Southwestern, Dallas, TX \\ George Dreszer, MD, MS, \\ Department of Plastic and Hand Surgery, Regions Hospital, St Paul, MN \\ Christine Fisher, MD, \\ Justin W. Zumsteg, MD, \\ Section of Plastic Surgery, University of Michigan, Ann Arbor, MI \\ Reda M. Jaber, BS, \\ Section of Plastic Surgery, University of Michigan, Ann Arbor, MI \\ Jennifer B. Hamill, MPH, \\ JBH Consulting, Shohola, PA
}

Division of Plastic and Reconstructive Surgery, University of Pittsburgh, Pittsburgh, Pennsylvania

Keith M. Hume, MA,

American Society of Plastic Surgeons, Arlington Heights, IL

\author{
J. Peter Rubin, MD, \\ Division of Plastic and Reconstructive Surgery, University of Pittsburgh, Pittsburgh, PA \\ Peter C. Neligan, MB, FRCS(I), FRCSC, FACS, \\ Division of Plastic Surgery, University of Washington, Seattle, WA \\ Loree K. Kallianen, MD, FACS, \\ Department of Plastic and Hand Surgery, Regions Hospital, St. Paul, MN
}

Ronald E. Hoxworth, MD,

Department of Plastic Surgery, University of Texas-Southwestern, Dallas, TX

Andrea L. Pusic, MD, MHS, FRCSC, and

Plastic and Reconstructive Surgery Service, Memorial Sloan Kettering Cancer Center, New York, NY

Edwin G. Wilkins, MD, MS

Section of Plastic Surgery, University of Michigan, Ann Arbor, MI

Disclosure Information: Nothing to disclose.

Abstract presented at the 2010 American Venous Forum meeting, 2010 American Association of Plastic Surgeons meeting, and 2010 Plastic Surgery Research Council.

Publisher's Disclaimer: This is a PDF file of an unedited manuscript that has been accepted for publication. As a service to our customers we are providing this early version of the manuscript. The manuscript will undergo copyediting, typesetting, and review of the resulting proof before it is published in its final citable form. Please note that during the production process errors may be discovered which could affect the content, and all legal disclaimers that apply to the journal pertain. 


\section{Abstract}

Background-The VTEPS Network is a consortium of five tertiary referral centers established to examine venous thromboembolism in plastic surgery patients. We report our mid-term analyses of the study's control group to 1) evaluate the incidence of VTE in patients who receive no chemoprophylaxis and 2) validate the Caprini Risk Assessment Model (RAM) in plastic surgery patients.

Study Design-Medical record review was performed at VTEPS centers for all eligible plastic surgery patients between March 2006 and June 2009. Inclusion criteria were Caprini score $\geq 3$, surgery under general anesthesia, and post-operative hospital admission. Patients who received chemoprophylaxis were excluded. Dependent variables included symptomatic DVT or PE within the first 60 post-operative days and time to DVT or PE.

Results-We identified 1126 historic control patients. The overall VTE incidence was $1.69 \%$. Approximately one in nine (11.3\%) patients with Caprini score $>8$ had a VTE event. Patients with Caprini score $>8$ were significantly more likely to develop VTE when compared to patients with Caprini score of 3-4 (OR 20.9, $\mathrm{p}<0.001$ ), 5-6 (OR 9.9, $\mathrm{p}<0.001$ ), or 7-8 (OR 4.6, p=0.015). Among patients with Caprini score 7-8 or Caprini score $>8$, VTE risk was not limited to the immediate post-operative period.

Conclusions-The Caprini RAM effectively risk-stratifies plastic and reconstructive surgery patients for VTE risk. Among patients with Caprini score $>8,11.3 \%$ have a post-operative VTE when chemoprophylaxis is not provided. In higher risk patients, there was no evidence that VTE risk is limited to the immediate post-operative period.

\section{INTRODUCTION}

Venous thromboembolism (VTE) is a disorder with short-term mortality and long-term morbidity. VTE has been deemed a major threat to patient safety by policymakers and payers, including the US Surgeon General (1), the Centers for Medicare and Medicaid Services (2), and the National Quality Forum (3). To fully identify VTE risk in surgical patients, recent publications advocate individualized patient risk assessment (4-7). The Caprini Risk Assessment Model (RAM) was derived over a decade ago based on a combination of clinical experience and published data (8-10). More recently, the Caprini RAM has been validated for 30-day VTE events in a large series of general, urology, and vascular surgery patients (5). Revised versions of the model have also been validated in post-bariatric body contouring patients (7) and medical inpatients $(11,12)$.

Plastic and reconstructive surgery patients are known to be at high risk for venous thromboembolism after surgery. Symptomatic VTE occurs with high frequency after postbariatric body contouring surgery, including circumferential abdominoplasty $(7.7 \%)$, abdominoplasty (5.0\%), and breast or upper body contouring (2.9\%) procedures (7). Using the modified Davison-Caprini RAM (13), Seruya and colleagues showed a 7.5\% VTE incidence in patients stratified to the "highest risk" group (14). Symptomatic, post-operative VTE occurs in $2.2 \%$ of women having flap-based breast reconstruction after mastectomy (15). However, asymptomatic VTE rates in the flap-based breast reconstruction population may be much higher. A recent study screened asymptomatic women prior to discharge using duplex ultrasound and demonstrated that $4 \%$ had occult DVT (16). In addition, a small case series demonstrated that $16.7 \%$ of women may have occult PE within 3 days of surgery (17).

The Venous Thromboembolism Prevention Study (VTEPS) was funded by the Plastic Surgery Educational Foundation in 2008. The study's primary objective is to examine the effectiveness of post-operative enoxaparin (Lovenox ${ }^{\circledR}$, Sanofi Aventis) for prevention of symptomatic VTE events in a diverse population of adult plastic and reconstructive surgery 
patients. The study's control group is comprised of historic control patients who had plastic and reconstructive surgery but did not receive post-operative heparin, low-molecular weight heparin, anti-factor Xa medications, or warfarin (collectively referred to as "chemoprophylaxis").

In this initial analysis of the VTEPS data, we sought to examine VTE incidence and when VTE events occur after plastic surgery. In addition, we examined the ability of the Caprini RAM to risk-stratify plastic and reconstructive surgery patients. Analyses were limited to VTEPS control patients, none of whom received chemoprophylaxis.

\section{METHODS}

\section{Study design}

VTEPS is being conducted at five tertiary care facilities in the United States. The analyses described below were limited to data from the VTEPS historic control group. Historic control patients were identified using medical record review for all plastic and reconstructive surgery cases performed at each of five VTEPS sites between January 2006 and June 2009. Historic control eligibility criteria included moderate to high risk for VTE (Caprini score $\geq 3$ ), operation under general anesthesia, and overnight hospital stay. Control patients did not receive heparin, low-molecular weight heparin (LMWH), factor Xa inhibitors, warfarin, or other means of prophylactic or therapeutic anti-coagulation for 60 days after surgery. This included the patient's inpatient stay and post-discharge course. Perioperative sequential compression devices were used.

\section{Independent variables}

At each study site, medical record review was performed by physician-led teams. Prior to chart review, each team participated in a standardized training session administered by the VTEPS study coordinators. Retrospective chart review was performed to identify VTE risk factors per the Caprini RAM (Figure 1). The factors were used to calculate a risk score based on risk factors present before (e.g. medical comorbidities or known thrombophilia) and during (e.g. surgery length or central venous line insertion) hospitalization. Additional independent variables included the year the procedure was performed, VTEPS site, patient gender, total number of operations, description of surgical procedure, receipt of chemoprophylaxis, administration of aspirin or clopidogrel, and length of hospitalization.

\section{Dependent variables}

Dependent variables of interest were identified using medical record review, including documentation from the operating room, inpatient stay, and outpatient visits. Records were reviewed for sixty days after surgery. Patients who lacked sixty day followup were excluded. Chart review identified symptomatic deep venous thrombosis, symptomatic pulmonary emboli, or hematoma requiring a second operation. All VTE events required confirmation using objective imaging (lower extremity venous duplex ultrasound, ventilation-perfusion scan, or pulmonary embolism protocol CT scan). Autopsy-proven DVT or PE were considered as positive outcomes when they were felt to be the proximate cause of death. Among those patients with an outcome of interest, time to VTE and time to re-operative hematoma in days were identified as additional dependent variables.

\section{Data storage and analysis}

A secure, password-protected website was created through a modification of the American Society of Plastic Surgeons' Tracking Operations and Outcomes for Plastic Surgeons (TOPS) platform (http://tops.plasticsurgery.org). The website was developed and maintained by Outcomes, Inc. (Cambridge, MA). De-identified patient-level data for risk factors and 
outcomes was uploaded and stored on Outcomes, Inc.'s secure data server. For analysis purposes, de-identified data was extracted by the American Society of Plastic Surgeons and provided to study personnel in Microsoft Excel spreadsheets.

Data analysis was performed using Stata11 (StataCorp LP, College Station, Texas). A VTE variable, including patients with either DVT or PE, was created. Descriptive statistics which examined DVT, PE, and VTE incidence were generated. For VTE risk analysis, patients were stratified by Caprini score at accepted and published levels (Caprini scores of 3-4, 5-6, $7-8$, and $>8)(5,18)$. Descriptive statistics on VTE rate by stratified Caprini score were generated. Group differences were examined using logistic regression. A value of $\mathrm{p}<0.05$ was considered significant. Kaplan-Meier analysis using stratified Caprini score was performed to examine the number of VTE events over time. Hazard ratios were generated.

The Institutional Review Board at each VTEPS site approved this study.

\section{RESULTS}

A total of 1126 historic control patients were identified from five VTEPS network sites. No patient in this series received pre- or post-operative chemoprophylaxis. The majority (79\%) of patients were in the Caprini 3-4 or Caprini 5-6 groups (Figure 2).

At sixty days after surgery, the overall VTE incidence was 1.69\%. Overall DVT incidence was $1.26 \%$ and overall PE incidence was $0.89 \%$. Patients with both DVT and PE comprised $0.44 \%$ of the total number of patients.

VTE incidence increased dramatically with increased Caprini score (Figure 3). In patients with Caprini score $>8,11.3 \%$ had a symptomatic VTE event between post-operative days 0 and 60. Patients with Caprini score $>8$ were significantly more likely to develop VTE when compared to patients with Caprini score of 3-4 (OR 20.9, p<0.001), 5-6 (OR 9.9, p<0.001), or 7-8 (OR 4.6, $\mathrm{p}=0.015)$. Additionally, patients with Caprini score 7-8 were significantly more likely to develop VTE when compared to patients with Caprini score 3-4 (OR 4.5, $\mathrm{p}=0.04)$ (Table 1).

Two of five sites systematically omitted data on time to VTE. These two sites did not provide the post-operative day on which VTE occurred for any patient with VTE at their institution. All data from these two sites were dropped prior to time series analysis. KaplanMeier analysis was performed on 1087 patients from the remaining three sites. Patients with Caprini score $>8$ had significantly increased hazard for VTE when compared to patients with Caprini score 3-4 (HR 48.6, p<0.001), 5-6 (HR 9.3, p=0.001), or 7-8 (HR 3.6, $\mathrm{p}=0.041$ ) (Table 2). In patients with Caprini score 7-8 and $>8$, VTE events occurred across the 60 day followup period (Figure 4).

\section{DISCUSSION}

Our data demonstrates that the Caprini RAM is a useful and effective tool to stratify plastic and reconstructive surgery patients for VTE risk. For patients with higher Caprini scores, a significantly greater likelihood of VTE events was observed. Approximately one in nine patients $(11.3 \%)$ with Caprini score $>8$ had a VTE event within 60 days after surgery. Particularly among patients with Caprini score $7-8$ or $>8$, there was no evidence that VTE risk is limited to the immediate post-operative period. 


\section{Venous thromboboembolism in plastic surgery patients}

Venous thromboembolic events carry the risk of both morbidity and mortality. Patients presenting with symptomatic PE have a $10 \%$ death rate within one hour. Of patients who survive, 50\% demonstrate evidence of right ventricular dysfunction. An additional 5\% will eventually develop chronic pulmonary hypertension (19). Between $4 \%$ and $7 \%$ of plastic surgeons report a patient death from post-operative PE (20,21). Deep venous thrombosis can damage venous valves, which can result in venous reflux and the post-thrombotic syndrome (PTS). Patients with PTS develop chronically swollen, tender, and ulcerated lower extremities. PTS is a major predictor of poor quality of life after DVT (22).

Plastic surgeons under-utilize chemoprophylaxis in high-risk patients and may fail to recognize risk factors when present $(20,21,23,24)$. Others may recognize risk factors but fail to actively modify them prior to surgery (25). Recently, plastic surgeons have been inundated with information on post-operative thromboembolic complications. Multiple recent publications have defined VTE incidence and risk factors in plastic surgery patients $(7,14,15,17,26-33)$. Barriers to surgeons providing chemoprophylaxis and methods to minimize VTE risk have been discussed in several letters to the editor (34-36). A modified version of the Caprini RAM has been validated in post-bariatric body contouring patients (7). However, no RAM had previously been validated in a broad range of adult plastic and reconstructive surgery patients.

\section{Validation of the Caprini Risk Assessment Model}

Prior versions of the Caprini RAM (9) placed all patients with a score $\geq 5$ into the same "highest risk" group. This designation was challenged by a recent publication by Bahl and colleagues from the University of Michigan (5). In a series of over 8000 general, urology, and vascular surgery patients, widely variable VTE rates were seen among patients previously lumped into the same "highest risk" category. Observed differences in VTE incidence among patients with Caprini score 5-6 (1.3\%), 7-8 (2.6\%) and >8 (6.5\%) were statistically significant. As a result, recent modifications of the Caprini RAM recognize patients with Caprini score $>8$ as a separate, "super high-risk" group. Extended duration chemoprophylaxis for 30 days after surgery is recommended for this "super high-risk" patient subgroup $(18,37)$.

Superficially, the study design for VTEPS and Bahl's analyses appear similar. Both used the Caprini RAM to retrospectively risk-stratify surgical patients and then examined VTE incidence by stratified Caprini score. However, the observed VTE incidence by stratified Caprini score was notably different between the VTEPS and Bahl patient populations. VTE incidence among "super high-risk" patients included in VTEPS approached twice the incidence seen in Bahl's patient population (11.3\% vs. $6.5 \%)$.

Plastic surgery patients are not necessarily at increased risk for VTE events when compared to general, urology, and vascular surgery patients. The differences in observed VTE incidence between the two studies may be explained by differences in study methodology. First, the VTEPS dataset contains 60 day patient followup, compared to 30 day outcomes in the Bahl study. Second, VTEPS methodology used manual chart review to identify VTE events; this method may be more robust than using hospital billing data. Finally, $32 \%$ of Bahl's Caprini $>8$ group received LMWH prophylaxis. In contrast, this analysis of the VTEPS database specifically excluded patients who received chemoprophylaxis.

\section{Late venous thromboembolism risk}

For patients with Caprini score 7-8 and >8, VTE risk was not limited to the immediate postoperative period. Our results echo recently published data from the UK's Million 
Women Study (38). The Million Women Study demonstrated that in middle-aged women, VTE risk may remain substantially elevated for at least 90 days after surgery. Published guidelines for total hip or knee replacement and hip fracture surgery support post-discharge chemoprophylaxis for up to 5 weeks after surgery (39). Similar recommendations for extended duration chemoprophylaxis have been made in selected groups of medical patients (40) and general surgery patients (18,41-43). The ENOXACAN II study was a randomized control trial examining 7 vs. 28 days of LMWH for VTE prevention after surgery for intraabdominal or pelvic malignancy. Extended-duration prophylaxis significantly reduced VTE events at 30 and 90 days after surgery (43). Interestingly, plastic surgery patients have demonstrated excellent compliance with outpatient LMWH injections for VTE prophylaxis. A small case series of post-discharge subcutaneous LMWH prophylaxis has shown $>90 \%$ medication compliance (14).

\section{Limitations}

Our analysis is limited by several factors. The VTEPS data was identified and uploaded in a retrospective manner. Risk factors were identified using physician review of the medical record. Thus, inadvertent omissions from the dictated medical record may have resulted in underestimation of a patient's Caprini risk score.

Multiple physicians and physician-led teams at five separate VTEPS sites identified and uploaded data to the study's central website. Sites contributed variable numbers of patients to the two study cohorts (historic control and clinical protocol). Inter-site variability in data collection may have been present. Prior to undertaking VTEPS, we attempted to control for this confounder. Each individual involved in data collection was provided with explicit, written study protocols. All individuals who collected VTEPS data participated in a standardized training session, administered by study personnel. Data was retrospectively checked by VTEPS core investigators for completeness. Ongoing feedback was provided to each VTEPS site. Incomplete data was dropped from the analysis.

Medical record review was conducted at each VTEPS site. Patients who had a VTE event diagnosed and/or treated at another hospital may not be represented in the VTEPS database. Literature published after our study protocol was designed and implemented supports elevated VTE risk for at least 90 days after surgery (38). However, patients whose VTE event occurred after post-operative day 60 are not included in our database. Clinical diagnosis of DVT and PE are known to be unreliable. Clinical examination alone is known to under-estimate true incidence of VTE (44-48). We did not screen patients for asymptomatic VTE, as has been performed in other high-risk surgical groups $(16,17,49,50)$. As a result of these factors, the VTEPS database likely under-represents the true incidence of VTE after plastic surgery.

\section{CONCLUSIONS}

The Caprini RAM effectively risk-stratifies plastic and reconstructive surgery patients for perioperative VTE risk. Among patients with Caprini score $>8,11.3 \%$ have a post-operative VTE when chemoprophylaxis is not provided. In patients with Caprini score 7-8 or Caprini score $>8$, there was no evidence that VTE risk is limited to the immediate post-operative period.

\section{Acknowledgments}

This study was funded by the Plastic Surgery Educational Foundation. Dr Pannucci receives salary support through NIH grant T32 GM-08616. 


\section{References}

1. Wakefield TW, McLafferty RB, Lohr JM, et al. Call to action to prevent venous thromboembolism. J Vasc Surg 2009;49:1620-1623. [PubMed: 19497526]

2. Centers for Medicare and Medicaid Services. press release, April 14 2008. http://www.cms.hhs.gov

3. National Quality Forum. press release, May 15 2008. http://www.qualityforum.org

4. Green D. VTE prophylaxis in aesthetic surgery patients. Aesth Surg J 2006;26:317-324.

5. Bahl V, Hu HM, Henke PK, et al. A validation study of a retrospective venous thromboembolism risk scoring method. Ann Surg 2009 Sep 22;241:344-50.

6. Agnelli G, Bolis G, Capussotti L, et al. A clinical outcome-based prospective study on venous thromboembolism after cancer surgery: The @ RISTOS project. Ann Surg 2006;243:89-95. [PubMed: 16371741]

7. Hatef DA, Kenkel JM, Nguyen MQ, et al. Thromboembolic risk assessment and the efficacy of enoxaparin prophylaxis in excisional body contouring surgery. Plast Reconstr Surg 2008;122:269_ 279. [PubMed: 18594417]

8. Motykie GD, Zebala LP, Caprini JA, et al. A guide to venous thromboembolism risk factor assessment. J Thromb Thrombolysis 2000;9:253-262. [PubMed: 10728025]

9. Caprini JA. Thrombosis risk assessment as a guide to quality patient care. Dis Mon 2005;51:70-78. [PubMed: 15900257]

10. Caprini JA, Arcelus JI, Reyna JJ. Effective risk stratification of surgical and nonsurgical patients for venous thromboembolic disease. Semin Hematol 2001;38:12-19. [PubMed: 11449339]

11. Arcelus JI, Candocia S, Traverso CI, et al. Venous thromboembolism prophylaxis and risk assessment in medical patients. Semin Thromb Hemost 1991;17:313-318. [PubMed: 1754887]

12. Zakai NA, Wright J, Cushman M. Risk factors for venous thrombosis in medical inpatients: Validation of a thrombosis risk score. J Thromb Haemost 2004;2:2156-2161. [PubMed: 15613021]

13. Davison SP, Venturi ML, Attinger CE, et al. Prevention of venous thromboembolism in the plastic surgery patient. Plast Reconstr Surg 2004;114:43E-51E.

14. Seruya M, Venturi ML, Iorio ML, Davison SP. Efficacy and safety of venous thromboembolism prophylaxis in highest risk plastic surgery patients. Plast Reconstr Surg 2008;122:1701-1708. [PubMed: 19050522]

15. Pannucci CJ, Chang EY, Wilkins EG. Venous thromboembolic disease in autogenous breast reconstruction. Ann Plast Surg 2009;63:34-38. [PubMed: 19546669]

16. Lemaine, V.; Mehara, BJ.; Pusic, AL., et al. Venous thromboembolism after microsurgical breast reconstruction: an objective analysis in 100 consecutive patients using low molecular weight heparin prophylaxis. Podium presentation, American Society of Plastic Surgeons meeting; 2009.

17. Kim EK, Eom JS, Ahn SH, et al. The efficacy of prophylactic low-molecular-weight heparin to prevent pulmonary thromboembolism in immediate breast reconstruction using the TRAM flap. Plast Reconstr Surg 2009;123:9-12. [PubMed: 19116509]

18. Caprini JA. Risk assessment as a guide for the prevention of the many faces of venous thromboembolism. Am J Surg 2010;199:S3-10. [PubMed: 20103082]

19. Kearon C. Natural history of venous thromboembolism. Circulation 2003 Jun 17;107:122-130.

20. Pannucci CJ, Oppenheimer AO, Wilkins EG. Practice patterns in venous thromboembolism prophylaxis: a survey of 606 reconstructive breast surgeons. Ann Plast Surg 2010;64:732-7. [PubMed: 20489401]

21. Clavijo-Alvarez JA, Pannucci CJ, Oppenheimer AO, et al. Prevention of venous thromboembolism (VTE) in body contouring surgery. A national survey of ASPS surgeons. Ann Plast Surg. In Press.

22. Kahn SR, Shbaklo H, Lamping DL, et al. Determinants of health-related quality of life during the 2 years following deep vein thrombosis. J Thromb Haemost 2008;6:1105-1112. [PubMed: 18466316]

23. Broughton G 2nd, Rios JL, Rohrich RJ, Brown SA. Deep venous thrombosis prophylaxis practice and treatment strategies among plastic surgeons: Survey results. Plast Reconstr Surg 2007;119:157-174. [PubMed: 17255670] 
24. Spring MA, Gutowski KA. Venous thromboembolism in plastic surgery patients: survey results of plastic surgeons. Aesth Surg J 2006;26:522-529.

25. Johnson RL, Hemington-Gorse SJ, Dhital SK. Do cosmetic surgeons consider estrogen-containing drugs to be of significant risk in the development of thromboembolism? Aesthetic Plast Surg 2008;32:743-747. [PubMed: 18446403]

26. Chen CM, Disa JJ, Cordeiro PG, et al. The incidence of venous thromboembolism after oncologic head and neck reconstruction. Ann Plast Surg 2008;60:476-479. [PubMed: 18434817]

27. Davison SP, Kessler CM, Al-Attar A. Microvascular free flap failure caused by unrecognized hypercoagulability. Plast Reconstr Surg 2009;124:490-495. [PubMed: 19644264]

28. Keyes GR, Singer R, Iverson RE, et al. Mortality in outpatient surgery. Plast Reconstr Surg 2008;122:245-250. discussion 251-253. [PubMed: 18594412]

29. Liao EC, Taghinia AH, Nguyen LP, et al. Incidence of hematoma complication with heparin venous thrombosis prophylaxis after TRAM flap breast reconstruction. Plast Reconstr Surg 2008;121:1101-1107. [PubMed: 18349626]

30. Miszkiewicz K, Perreault I, Landes G, et al. Venous thromboembolism in plastic surgery: Incidence, current practice and recommendations. J Plast Reconstr Aesthet Surg 2009;62:580-588. [PubMed: 19264567]

31. Murray DJ, Neligan PC, Novak CB, et al. Free tissue transfer and deep vein thrombosis. J Plast Reconstr Aesthet Surg 2008;61:687-692. [PubMed: 18207471]

32. Patronella CK, Ruiz-Razura A, Newall G, et al. Thromboembolism in high-risk aesthetic surgery: Experience with 17 patients in a review of 3871 consecutive cases. Aesthet Surg J 2008;28:648655. [PubMed: 19083593]

33. Seruya M, Baker SB. MOC-PS(SM) CME article: Venous thromboembolism prophylaxis in plastic surgery patients. Plast Reconstr Surg 2008;122:1-9. [PubMed: 18698237]

34. Davison SP, Massoumi W. Our complication, your problem. Plast Reconstr Surg 2007;120:14281429. [PubMed: 17898624]

35. Ersek RA. General anesthesia gases are a common denominator in cases of thromboembolism. Aesthet Surg J 2009;29:340-341. [PubMed: 19717073]

36. Pannucci CJ, Wilkins EG. Hematoma risk should not preclude the use of venous thromboembolism prophylaxis. Aesthet Surg J 2009;29:338. author reply 339. [PubMed: 19717071]

37. Henke PH, Pannucci CJ. Venous thromboembolism risk factor assessment and prophylaxis. Phlebology. In Press.

38. Sweetland S, Green J, Liu B, et al. Duration and magnitude of the postoperative risk of venous thromboembolism in middle aged women: Prospective cohort study. BMJ 2009;339:b4583. [PubMed: 19959589]

39. Geerts WH, Bergqvist D, Pineo GF, et al. Prevention of venous thromboembolism: American College of Chest Physicians evidence-based clinical practice guidelines. Chest (8th) 2008;133:381S-453S. [PubMed: 18574271]

40. Yale SH, Medlin SC, Liang H, et al. Risk assessment model for venothromboembolism in posthospitalized patients. Int Angiol 2005;24:250-254. [PubMed: 16158034]

41. Rasmussen MS. Preventing thromboembolic complications in cancer patients after surgery: A role for prolonged thromboprophylaxis. Cancer Treat Rev 2002;28:141-144. [PubMed: 12234565]

42. Rasmussen MS, Jorgensen LN, Wille-Jorgensen P, et al. Prolonged prophylaxis with dalteparin to prevent late thromboembolic complications in patients undergoing major abdominal surgery: A multicenter randomized open-label study. J Thromb Haemost 2006;4:2384-2390. [PubMed: 16881934]

43. Bergqvist D, Agnelli G, Cohen AT, et al. Duration of prophylaxis against venous thromboembolism with enoxaparin after surgery for cancer. N Engl J Med 2002;346:975-980. [PubMed: 11919306]

44. Mclachlin J, Richards T, Paterson JC. An evaluation of clinical signs in the diagnosis of venous thrombosis. Arch Surg 1962;85:738-744. [PubMed: 13932125]

45. Haeger K. Problems of acute deep venous thromosis. II. mobilization and discharge of the patient. Angiology 1969;20:280-286. [PubMed: 4182321] 
46. Goldhaber SZ, Hennekens CH, Evans DA, et al. Factors associated with correct antemortem diagnosis of major pulmonary embolism. Am J Med 1982;73:822-826. [PubMed: 7148876]

47. Douketis JD, Kearon C, Bates S, et al. Risk of fatal pulmonary embolism in patients with treated venous thromboembolism. JAMA 1998;279:458-462. [PubMed: 9466640]

48. Anderson FA Jr, Wheeler HB, Goldberg RJ, et al. A population-based perspective of the hospital incidence and case-fatality rates of deep vein thrombosis and pulmonary embolism. the worcester DVT study. Arch Intern Med 1991;151:933-938. [PubMed: 2025141]

49. Wahl WL, Brandt MM, Ahrns KS, et al. Venous thrombosis incidence in burn patients: Preliminary results of a prospective study. J Burn Care Rehabil 2002;23:97-102. [PubMed: 11882798]

50. Wibbenmeyer LA, Hoballah JJ, Amelon MJ, et al. The prevalence of venous thromboembolism of the lower extremity among thermally injured patients determined by duplex sonography. J Trauma 2003;55:1162-1167. [PubMed: 14676666] 


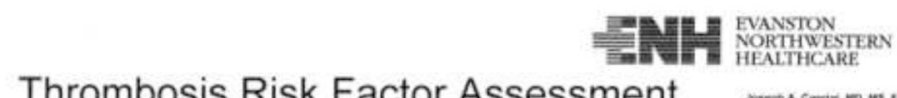

\title{
Thrombosis Risk Factor Assessment
}

\author{
Patient's Name:
} Age: __ Sex:

Wgt: Ibs

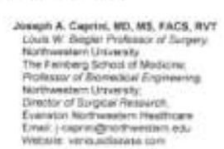

Choose All That Apply

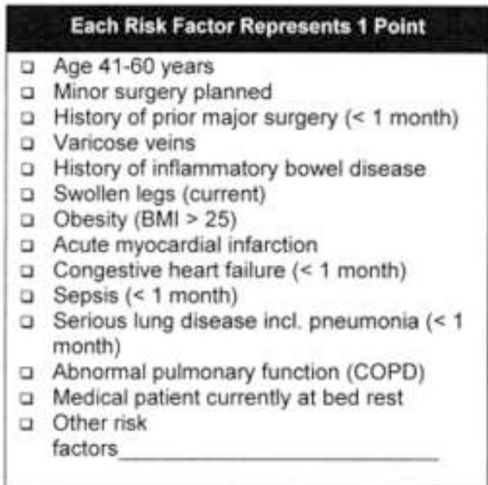

Each Risk Factor Represents 3 Points

a Age over 75 years

a History of DVT/PE

a Family history of thrombosis*

a Positive Factor $V$ Leiden

a Positive Prothrombin 20210A

a Elevated serum homocysteine

a Positive lupus anticoagulant

- Elevated anticardiolipin antibodies

a Heparin-induced thrombocytopenia (HIT)

a Other congenital or acquired thrombophilia

If yes:

Type

'most frequently missed risk factor
Each Risk Factor Represents 2 Points

a Age 60-74 years

a Arthroscopic surgery

a Malignancy (present or previous)

a Major surgery (> 45 minutes)

a Laparoscopic surgery (> 45 minutes)

a Patient confined to bed ( $>72$ hours)

a Immobilizing plaster cast (< 1 month)

Central venous access

\section{Each Risk Factor Represents 5 Points}

a Elective major lower extremity arthroplasty

a Hip, pelvis or leg fracture ( $<1$ month)

a Stroke (< 1 month)

a Multiple trauma (<1 month)

- Acute spinal cord injury (paralysis) $(<1$ month)

\section{For Women Only (Each Ropresents 1 Point)}

a Oral contraceptives or hormone replacement therapy

a Pregnancy or postpartum ( $<1$ month)

a History of unexplained stillborn infant, recurrent spontaneous abortion $(\geq 3)$. premature birth with toxemia or growthrestricted infant

\section{Total Risk Factor Score}

Figure 1.

The Caprini Risk Assessment Model. (Adapted from: Caprini JA. Thrombosis risk assessment as a guide to quality patient care. Dis Mon 2005;51:70-78 with permission.) 


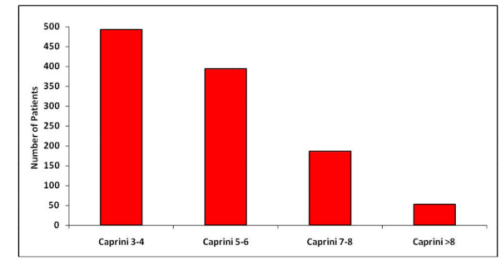

Figure 2.

Histogram of Caprini score in plastic and reconstructive surgery patients $(n=1,126)$. 


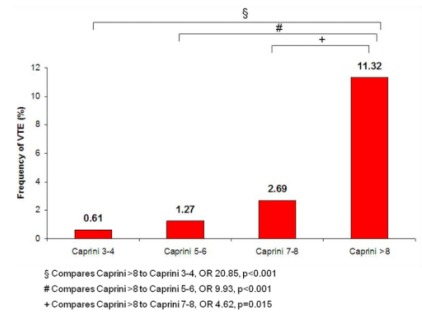

Figure 3.

Rates of VTE in the first 60 post-operative days by stratified Caprini score $(n=1,126)$. 


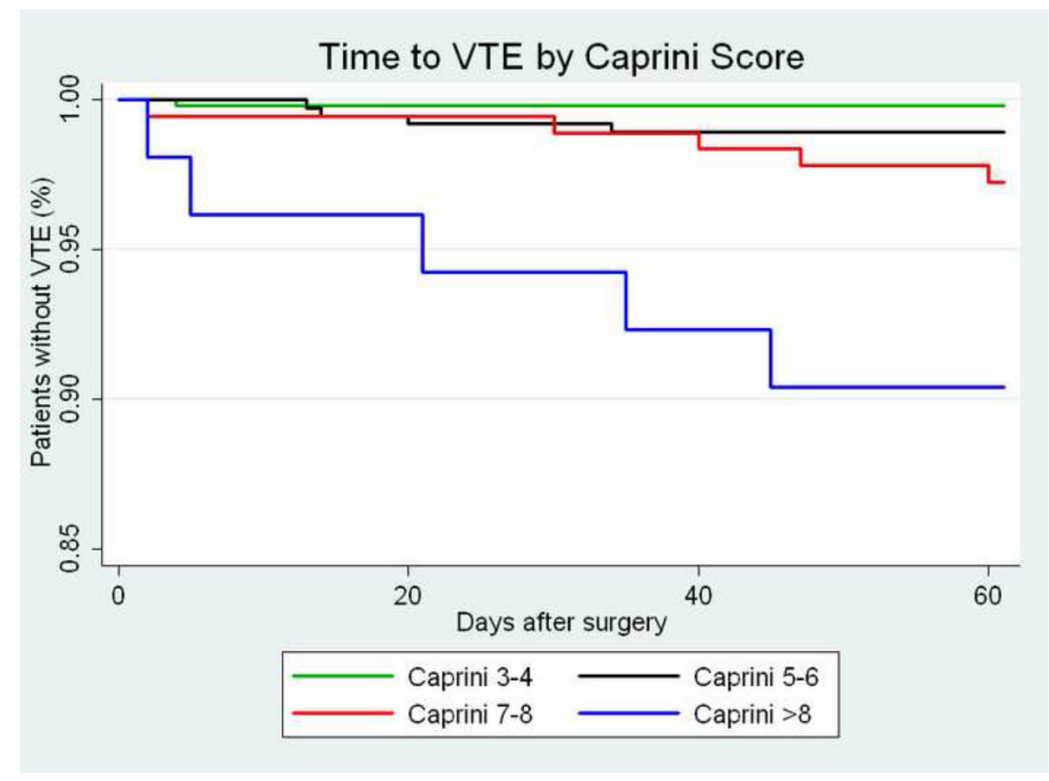

Figure 4.

Survival analysis examining time to VTE by stratified Caprini score $(n=1,087)$. 


\section{Table 1}

Sixty-day Odds for VTE Stratified by Caprini Score

\begin{tabular}{llll}
\hline & Caprini 5-6 & Caprini 7-8 & Caprini $>8$ \\
\hline Caprini 3-4 & $2.1(0.5-8.8)$ & $4.5(1.1-19.1)$ & $20.9(5.1-86.1)$ \\
& $\mathrm{p}=0.312$ & $\mathrm{p}=0.04$ & $\mathrm{p}<0.001$ \\
Caprini 5-6 & -- & $2.2(0.6-7.5)$ & $9.9(2.9-33.8)$ \\
& & $\mathrm{p}=0.231$ & $\mathrm{p}<0.001$ \\
Caprini 7-8 & -- & -- & $4.6(1.4-15.8)$ \\
& & & $\mathrm{p}=0.015$ \\
\hline
\end{tabular}

Data are presented as odds ratio (95\% confidence interval). 


\section{Table 2}

Sixty-day Hazard for VTE Stratified by Caprini Score

\begin{tabular}{llll}
\hline & Caprini 5-6 & Caprini 7-8 & Caprini $>8$ \\
\hline Caprini 3-4 & $5.2(0.6-46.7)$ & $13.3(1.6-114.1)$ & $48.6(5.7-416.0)$ \\
& $\mathrm{p}=0.139$ & $\mathrm{p}=0.018$ & $\mathrm{p}<0.001$ \\
Caprini 5-6 & -- & $2.6(0.7-9.5)$ & $9.3(2.5-34.7)$ \\
& & $\mathrm{p}=0.162$ & $\mathrm{p}=0.001$ \\
Caprini 7-8 & -- & -- & $3.6(1.1-12.6)$ \\
& & & $\mathrm{p}=0.041$ \\
\hline
\end{tabular}

Data are presented as hazard ratio ( $95 \%$ confidence interval). 